\title{
Tetraspanin 1 promotes invasiveness of cervical cancer cells
}

\author{
SEBASTIAN HÖLTERS ${ }^{1}$, JELENA ANACKER ${ }^{4}$, LARS JANSEN $^{1}$, \\ KATRIN BEER-GRONDKE ${ }^{1}$, MATTHIAS DÜRST ${ }^{1}$ and IGNACIO RUBIO ${ }^{2,3}$ \\ ${ }^{1}$ Department of Gynecology and Obstretrics, ${ }^{2}$ Center for Molecular Biomedicine, Institute of Molecular Cell Biology, \\ ${ }^{3}$ Center for Sepsis Control and Care (CSCC), Jena University Hospital, Jena; ${ }^{4}$ Department of Obstretrics \\ and Gynecology, University of Würzburg, Würzburg, Germany
}

Received March 5, 2013; Accepted April 22, 2013

DOI: 10.3892/ijo.2013.1980

\begin{abstract}
Tetraspanins are a heterogeneous group of 4-transmembrane proteins that segregate into so-called tetraspanin-enriched microdomains (TEMs) along with other cell surface proteins such as integrins. TEMs of various types are reportedly involved in the regulation of cell growth, migration and invasion of several tumour cell types, both as suppressors or supporting structures. Tetraspanin 1 (Tspan1, NET-1), a member of the transmembrane 4 superfamily (TM4SF) of tetraspanins, is overexpressed in high-grade cervical intraepithelial neoplasia (CIN) and terminal carcinomas but its precise function in the context of carcinoma of the cervix uteri is not known. Here, we present a comprehensive investigation of the role of tetraspanin 1 in the cervical cancer cell lines SiHa and HeLa. We document that tetraspanin 1 increases the invasive potential of cervical cancer cells, whereas proliferation, growth in soft agar and adhesion are largely unaffected. In line with the latter findings, our data exclude the participation of testraspanin in integrin-mediated activation of focal adhesion kinase (FAK), paxillin and phosphoinositide-3-kinase (PI3K) and in EGFR-dependent signalling to the Ras/Erk pathway. In conclusion, our data argue against a role for tetraspanin 1 as a genuine mediator of cell surface receptor signalling but rather document a role for tetraspanin 1 in the control of cervical cancer cell motility and invasion.
\end{abstract}

\section{Introduction}

Cervical cancer is the third most common cancer among women worldwide (1) emerging from pre-malignant precursor lesions that are gradually referred to as cervical intraepithelial neoplasia (CIN), graded mild dysplasia (CIN I), moderate

Correspondence to: Dr Ignacio Rubio, Institute of Molecular Cell Biology, Center for Molecular Biomedicine, University Hospital, Friedrich-Schiller-University, Hans-Knöll-Str. 2, D-07745 Jena, Germany

E-mail: ignacio.rubio@med.uni-jena.de

Key words: tetraspanin 1, Tspan1, NET-1, carcinoma of the cervix, cervical intraepithelial neoplasia, cervical cancer, tumour invasion dysplasia (CIN II) and severe dysplasia/carcinoma in situ (CIN III). Cervical cancer shows a prevalence of human papillomaviruses (HPV) of $99.7 \%$ (2). For humans, approximately 100 HPV-types have been described (3), approximately 40 of which infect the anogenital tract (4), the most common carcinogenic types being HPV16, 18, 31 and 45 (2,5,6). The HPV life cycle requires infection of proliferating epithelial cells and the 'early' proteins E5, E6 and E7 are known to foster proliferation (6). During cervical cancer development, the HPV genome may integrate into the host genome, whereby parts of the HPV genome, including the E5 gene, are deleted (7). The oncoproteins E6 and E7 can immortalize cells independently of each other but transformation efficiency in the presence of both is increased (8-11). Finally, during differentiation 'late' genes are expressed in the upper layers of the mucosa and viral particles are produced and released (12). Despite this rather precise understanding of the aetiology of carcinoma of the cervix, tumour classification is often arduous to accomplish and there is still a manifested need for better diagnostic and prognostic tools.

Tetraspanins represent a superfamily of transmembrane proteins with 33 members in humans (13) that was first described in studies of tumour associated proteins $(14,15)$. Tetraspanins are glycoproteins that consist of four transmembrane domains, forming two hydrophilic extracellular domains as well as a small intracellular loop. Both termini, carboxyl and amino, are cytoplasmatic and the extracellular domains contain several glycosylation sites (16-19). Tetraspanins are discriminated from other integral 4-transmembrane proteins by virtue of a highly conserved 4-amino acid motif within the large extracellular domain and conserved cystein residues within each of the four transmembrane domains (19). The large extracellular domain consists of two subdomains that mediate tetraspanin-tetraspanin homophilic binding or interactions with other transmembrane proteins, respectively $(20,21)$. On their intracellular, membrane-proximal portion tetraspanins contain cysteines that undergo palmitoylation, a feature deemed to be important for protein-protein interactions (22). Tetraspanins are not only found at the plasma membrane (PM), they also associate with membranes of endo- and exosomes $(23,24)$. Thus, via their large extracellular domain and the intracellular palmitoylation moieties, tetraspanins are able to bind and thereby form complexes with each other and other transmembrane proteins, for instance members of the integrin 
family (reviewed in ref. 25). These tetraspanin network or tetraspanin enriched microdomains (TEM) contribute to the organisation of cell surface proteins and can influence signal transduction $(26,27)$.

The role of tetraspanins in carcinogenesis and cancer progression is probably multifaceted. For instance, levels of the tetraspanin CD151 are elevated in several types of cancer including high-grade breast cancer and poor-outcome endometrial cancer and correlate with decreased survival (28-31). Furthermore, CD151 is able to increase motility and invasiveness of the breast cancer cell line MDA-MB-231 and depletion of CD151 leads to reduced tumour progression in vivo (32). In contrast, tetraspanin CD9 inhibits cell motility and growth of the human lung adenocarcinoma cell line MAC10 and the myeloma cell line ARH77 (33). An inverse correlation of CD9 expression versus metastasis was documented for breast cancer (34) and reduction of CD9 expression correlates with poor prognosis in breast (35) or lung carcinoma (36). Beyond cancer, tetraspanins and TEMs are involved in viral diseases. CD82 plays a role in hepatitis $\mathrm{C}$ virus binding and infection (37). Similarly the participation of TEMs containing CD9, CD63, CD81 and CD82 has been reported in the context of HIV-1 infections (38). Recently, the involvement of TEMs including CD63 and CD151 in the course of HPV16 infection was documented (39).

The tetraspanin family member tetraspanin 1 (Tspan1, NET-1) is upregulated in human hepatocellular carcinoma (42), gastric carcinoma (43), colorectal adenocarcinoma (44) and ovarian carcinomas (45), suggesting that tetraspanin 1 may be involved in genesis and progression of various forms of neoplasias. In line with this possibility tetraspanin 1 is important for proliferation and invasion of various human carcinoma cell lines $(46,47)$. We have previously reported that tetraspanin 1 expression is markedly increased at the RNA and protein level in high-grade CIN and cervical cancer $(40,41)$, but its role in cervical cancer biology has remained obscure. In the present study we investigated the function of tetraspanin 1 in 2 cervical cancer cell lines, looking both at functional effects and the possible involvement of tetraspanin 1 in growth factor signal transduction. Our data argue against a function of tetraspanin 1 as a genuine signal transducing protein and rather highlight an effect on cervical cancer cell invasion processes.

\section{Materials and methods}

Reagents and antibodies. The antibodies used for western blotting and immunohistochemistry are listed in Table I. All antibodies except for Tspan1 were commercially available. For the generation of Tspan1 antibodies, the major extracellular domain of Tspan1 was expressed as a histidine tagged fusion protein in E. coli (41). Gel purified protein was injected intraperitoneally into Balb/c mice. The mice were boosted 2 and 4 weeks after immunization. Serum was collected 3 days after the last boost to assess the immune response by indirect immune-fluorescence on Tspan 1 transfected Cos-7 cells. Mouse splenocytes were fused with SP2/0 myeloma cells according to standard procedures. Hybridomas producing antibodies with reactivity to Tspan1 were selected by ELISA. Clone 4/12 was found to be suitable both for immunocyto- and immunohistochemistry. Secondary peroxidase-coupled antibodies were purchased from Dianova. EGF and poly-L-lysine were purchased from Sigma-Aldrich and extracellular matrix proteins (ECM)-Proteins fibronectin, laminin, collagen type I and III from Millipore.

Cell culture. SiHa and HeLa cells (ATCC) were cultured in DMEM (Gibco) supplemented with $10 \%$ fetal calf serum (FCS) (Sigma-Aldrich) under $37^{\circ} \mathrm{C}, 95 \%$ humidity and $5 \% \mathrm{CO}_{2}$ content. Experiments were done with cell passages 5-20.

Adenoviral transduction. Cells $\left(4 \times 10^{5}\right)$ seeded in $60 \mathrm{~mm}$ or $10^{6}$ cells in $100 \mathrm{~mm}$ dishes were infected with adenovirus particles coding for EGFP or tetraspanin 1, respectively, at a multiplicity of infection of 10 . Twenty-four hours later medium was replaced and cells were cultured additional $24 \mathrm{~h}$ and processed for further experiments. Transduction efficiency, as determined by fluorescence microscopy (EGFP) and immunocytochemistry with the monoclonal antibody clone 4/12 against tetraspanin 1, ranged between 80 and $90 \%$.

Matrix invasion. Cell invasion was determined using Matrigel coated cell culture inserts $(8-\mu \mathrm{m}$ pore size for 24 -well cell culture plates) (BD Biosciences, BD Falcon ${ }^{\mathrm{TM}}$ ) following the manufacturer's instructions. Briefly, Matrigel was diluted 1:3 in serum-free DMEM and $30 \mu \mathrm{l}$ diluted Matrigel were poured into each insert and allowed to gelatinise for $1 \mathrm{~h}$ at $37^{\circ} \mathrm{C}$. The upper chamber was filled with $10^{5}$ of untransduced, EGFP or tetraspanin 1 expressing SiHa cells in $200 \mu$ l growth medium (containing 10\% FCS) while the lower compartment contained $500 \mu \mathrm{l}$ growth medium. After $72 \mathrm{~h}$ cells at the lower membrane side were rinsed with double distilled water $\left(\mathrm{ddH}_{2} \mathrm{O}\right)$ and fixed with ice-cold $80 \%$ ethanol for $20 \mathrm{~min}$. Wells were washed with $\mathrm{ddH}_{2} \mathrm{O}$ and matrix material and remaining cells inside the insert were thoroughly removed with a cotton tip. The fixed cells were stained with $0.1 \%$ crystal violet for $5 \mathrm{~min}$ and washed once with $\mathrm{ddH}_{2} \mathrm{O}$. Stained cells were decolourised with $10 \%$ acetic acid and absorbance was measured at $630 \mathrm{~nm}$ (48). Four independent experiments with 4-fold determinations were carried out.

Proliferation assay. Untransduced, EGFP or tetraspanin 1 expressing $\mathrm{SiHa}$ cells $\left(2 \times 10^{4}\right)$ were seeded on a 24 -well cell culture plate. Cells were cultured for $24 \mathrm{~h}$ and deprived of serum for $6 \mathrm{~h}$. Medium was changed to DMEM supplemented with $10 \%$ FCS and proliferation was determined 6, 24, 48 and $72 \mathrm{~h}$ later by using the CellTiter $96^{\circledR}$ Non-Radioactive Cell Proliferation assay (Promega) following the manufacturer's protocol. For precise quantification, four independent experiments with duplicates for $\mathrm{SiHa}$ and five independent experiments with triplicates and duplicates for EGFP and tetraspanin 1 , respectively, were performed at $6 \mathrm{~h}$ after serum re-addition.

Colony formation assay. Agarose (0.5\%) (5 ml) in DMEM supplemented with FCS were added to each well of a 6-well cell culture plate and allowed to gelatinise for $3 \mathrm{~h}$ at room temperature. Agarose (0.3\%) (3 ml) in DMEM supplemented with $10 \%$ FCS and containing $5 \times 10^{4}$ untransduced, EGFP or tetraspanin 1 expressing $\mathrm{SiHa}$ cells was poured on top of 
Table I. Antibodies used in the present study.

\begin{tabular}{|c|c|c|c|c|}
\hline Protein & Antibody & Species/type & Dilution & Company \\
\hline \multirow[t]{2}{*}{ EGF-receptor } & $\alpha$-EGFR-IgG & Rabbit/polyclonal & $1: 1,000$ & Cell Signaling \\
\hline & $\alpha-p E G F R(Y 1068)-I g G$ & Rabbit/polyclonal & $1: 1,000$ & Invitrogen \\
\hline \multirow[t]{2}{*}{ Erk1/2 } & $\alpha$-Erk1/2-IgG & Rabbit/polyclonal & $1: 1,000$ & Cell Signaling \\
\hline & $\alpha-p E r k 1 / 2(\mathrm{~T} 202 / \mathrm{Y} 204)-\operatorname{IgG}$ & Mouse/monoclonal & $1: 1,000$ & Cell Signaling \\
\hline Focal & $\alpha-F A K-I g G$ & Rabbit/polyclonal & $1: 1,000$ & Cell Signaling \\
\hline Adhesion & $\alpha-p F A K(Y 397)-I g G$ & Rabbit/polyclonal & $1: 1,000$ & Cell Signaling \\
\hline \multirow[t]{2}{*}{ Kinase } & $\alpha-p F A K(Y 576 / 577)-I g G$ & Rabbit/polyclonal & $1: 1,000$ & Cell Signaling \\
\hline & $\alpha-p F A K(Y 925)-\operatorname{IgG}$ & Rabbit/polyclonal & $1: 1,000$ & Cell Signaling \\
\hline \multirow[t]{2}{*}{ Paxillin } & $\alpha-P a x i l l i n-I g G$ & Rabbit/polyclonal & $1: 1,000$ & Santa Cruz Biotechnology \\
\hline & $\alpha$-pPaxillin(Y118)-IgG & Rabbit/polyclonal & $1: 1,000$ & Cell Signaling \\
\hline \multirow[t]{4}{*}{ Ras } & $\alpha$-pan-Ras-IgG & Mouse/monoclonal & $1: 1,000$ & Calbiochem \\
\hline & $\alpha-\mathrm{K}-\mathrm{Ras}-\mathrm{IgG}$ & Mouse/monoclonal & $1: 1,000$ & Santa Cruz Biotechnology \\
\hline & $\alpha-\mathrm{N}$-Ras-IgG & Mouse/monoclonal & $1: 1,000$ & Santa Cruz Biotechnology \\
\hline & $\alpha-H-R a s-I g G$ & Mouse/monoclonal & $1: 1,000$ & Santa Cruz Biotechnology \\
\hline \multirow[t]{2}{*}{ Tetraspanin 1} & $\alpha-C 4.8 /$ NET-1-IgG \#4/12 & Mouse/monoclonal & $1: 1,000$ & Jena University Hospital \\
\hline & & & & Department of Gynecology \\
\hline \multirow[t]{2}{*}{$\mathrm{p} 16^{\mathrm{INK} 4 \mathrm{a}}$} & $\alpha-\mathrm{p} 16^{\mathrm{INK} 4 \mathrm{a}}-\mathrm{IgG}$ & Mouse/monoclonal & Ready to use CINtec ${ }^{\circledR}$ & MTM Laboratories \\
\hline & & & Histology kit & Heidelberg \\
\hline MMP14 & $\alpha$-MMP14-IgG & Rabbit/polyclonal & $1: 1,000$ & Abcam \\
\hline
\end{tabular}

the agarose layer. Cells were cultured over 3 weeks and foci were scored manually under a microscope. Four independent experiments with triplicates were done.

Cell adhesion. Cell culture plates (96-well) (BD Falcon ${ }^{\mathrm{TM}}, \mathrm{BD}$ Biosciences) were coated with $100 \mu \mathrm{l} /$ well of $150 \mu \mathrm{g} / \mathrm{ml}$ PolyL-lysine or $10 \mu \mathrm{g} / \mathrm{ml}$ fibronectin, laminin, collagen type I and III (Millipore), respectively. Proteins were diluted in sterile PBS and coated overnight at room temperature followed by blocking with $100 \mu \mathrm{l} /$ well $2 \%$ bovine serum albumin (BSA) in PBS for $2 \mathrm{~h}$ at room temperature and 2 washes with $200 \mu \mathrm{l} /$ well PBS. Untransduced, EGFP or tetraspanin 1 expressing SiHa cells $\left(2 \times 10^{4}\right)$ were added to each well and incubated for $2 \mathrm{~h}$. Wells were washed twice with PBS and cells were stained with $0.1 \%$ crystal violet for 5 min followed by 2 washes with PBS. Stained cells were de-stained with $10 \%$ acetic acid and absorbance was measured at $630 \mathrm{~nm}$. Four independent experiments with 4 -fold determination were done.

Immunohistochemistry and -cytochemistry. For immunohistochemistry, tissue arrays with paraffin embedded samples of normal cervical tissue, low- and high-grade cervical lesions and cervical cancer tissues were stained with a monoclonal antibody, clone 4/12, against tetraspanin 1 and antibodies against $\mathrm{p} 16^{\mathrm{INK} 4 \mathrm{a}}$. Signal detection was performed using EnVison $^{\mathrm{TM}}$ Detection System (Dako). For immunocytochemistry, adenovirally transduced $\mathrm{SiHa}$ cells were spotted on a glass slide and fixed with 4\% paraformaldehyde in TBS-Tween (50 mM Tris, $150 \mathrm{mM} \mathrm{NaCl}, 0.1 \%$ Tween-20). Fixed cells were incubated with $0.6 \%$ hydrogen peroxide for $7 \mathrm{~min}$ followed by 20 min blocking with goat serum, 1:5 diluted in TBS-Tween. Cells were incubated with monoclonal antibody against tetraspanin 1 for $1 \mathrm{~h}$ and bound antibodies were detected with the EnVison Detection System (Dako). Between every incubation step a 5 min wash step with TBS-Tween was implemented. All steps were done at room temperature.

Cell stimulation and western blotting. Growth factor stimulation: $4 \times 10^{5}$ cells seeded on $60 \mathrm{~mm}$ cell culture dishes were subjected to adenoviral transduction as described above and deprived of serum overnight prior to stimulation with $100 \mathrm{ng} /$ $\mathrm{ml}$ EGF. Cells were lysed in $500 \mu \mathrm{l}$ ice-cold lysis buffer: $50 \mathrm{mM}$ Tris, $50 \mathrm{mM} \mathrm{NaCl}, 5 \mathrm{mM} \mathrm{MgCl}{ }_{2}, 1 \mathrm{mM}$ EGTA, $1 \%$ NP-40, $10 \%$ glycerol, protease inhibitor mix HP (Serva) and phosphatase inhibitors. Extracts were cleared by centrifugation and the supernatants were processed by SDS-PAGE separation and western blotting using the antibodies listed in Table I.

Integrin activation: $60-\mathrm{mm}$ cell culture dishes were coated with matrix proteins as described before. Adenovirally transduced and untransduced cells were starved for $16 \mathrm{~h}$ in serum-free DMEM, detached by trypsinization and resuspended in serum-free DMEM supplemented with $10 \mu \mathrm{g} / \mathrm{ml}$ trypsin inhibitor (Gibco, Invitrogen) and $2 \% \mathrm{BSA}$. Cells were rotated for $1 \mathrm{~h}$ at $37^{\circ} \mathrm{C}$ and $3 \times 10^{5}$ cells in $1 \mathrm{ml}$ were added to 


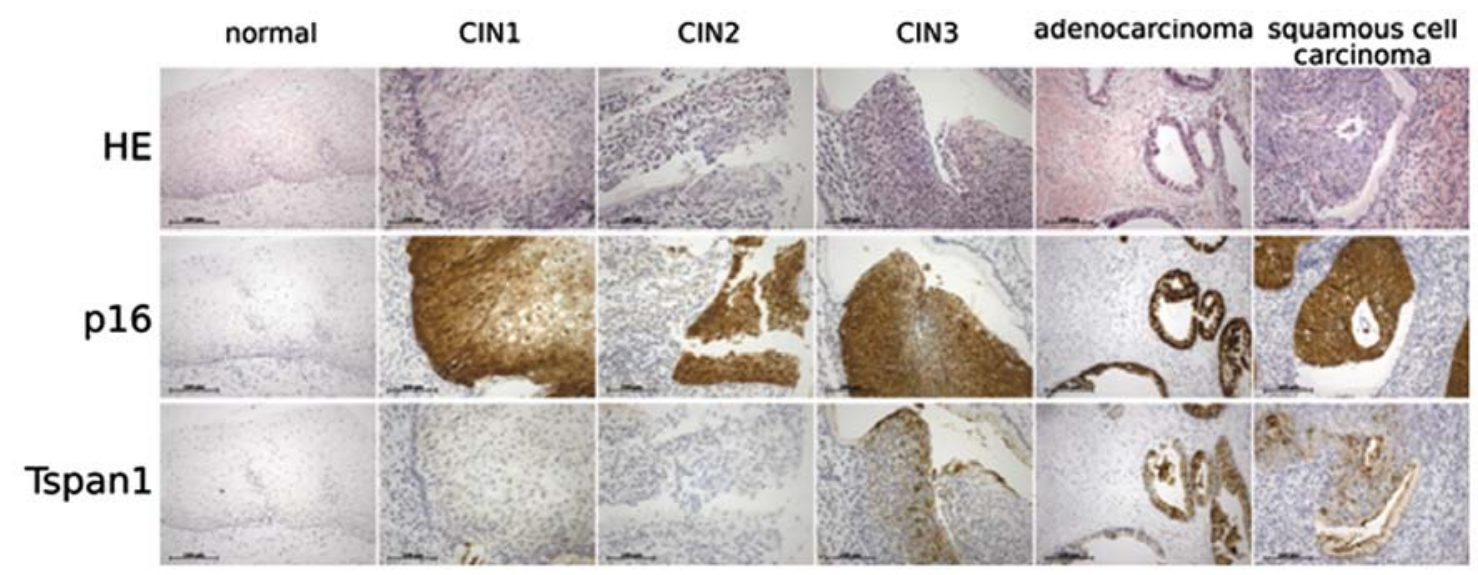

Figure 1. Tetraspanin 1 is highly expressed at the growth front of cervical cancer and CIN III. Tetraspanin 1 and p16 ${ }^{\text {INK4a }}$ staining of cervical cancer specimens of various clinical grades. Note that the tetraspanin 1-positive areas are located at growth fronts of the tumour or high-grade CIN.

each dish. Cells were cultivated for $2 \mathrm{~h}$ and lysed with $100 \mu \mathrm{l}$ ice-cold lysis solution: $50 \mathrm{mM}$ Tris; $150 \mathrm{mM} \mathrm{NaCl}, 5 \mathrm{mM}$ $\mathrm{MgCl}_{2}, 1 \mathrm{mM}$ EGTA, $1 \%$ NP-40, protease inhibitor Mix HP (Serva) and phosphatase inhibitors. Lysates were cleared by centrifugation and processed for SDS-PAGE and western blotting.

Expression of matrix metalloproteinases (MMPs). Expression of MMPs was analysed: i) by semiquantitative RT-PCR for one sample set (control, EGFP and tetraspanin 1 expressing $\mathrm{SiHa}$ and HeLa cells, respectively), ii) by western blotting of two sample sets, and iii) via immunohistochemistry with tissue arrays. Methods were described in detail previously (49).

Ethic statement. This study was approved by the ethics committee of the Friedrich-Schiller University Jena (reference number 175-02/00).

Statistical analysis. Statistical significance was determined by non-parametric, independent homogeneity tests Kruskal-Wallis test for more than two groups and Mann-Whitney test for two groups, using analysis software SPSS Statistics 19 (IBM).

\section{Results}

Tetraspanin 1 is upregulated at the growth front of CIN III and cervical cancer. We have previously reported that tetraspanin 1 expression becomes detectable earliest in highgrade cervical neoplasia and continues in cervical cancer $(40,41)$, but its precise role in cancer development remains unclear. To determine more accurately the expression pattern of tetraspanin 1 in cervical cancer, tissue arrays were analysed by immunohistochemistry using the newly generated monoclonal antibody \#4/12 (see Materials and methods). The arrays contained normal cervical tissues, low- and high-grade cervical lesions and cervical carcinoma tissue. As expected from our previous analyses using polyclonal antibodies, tetraspanin 1 expression was not detectable in normal cervical tissue and low-grade cervical intraepithelial lesions (CIN) I and II (Fig. 1 and data not shown). Expression of tetraspanin 1 was, however, extensive in high-grade CIN III and cervical
A

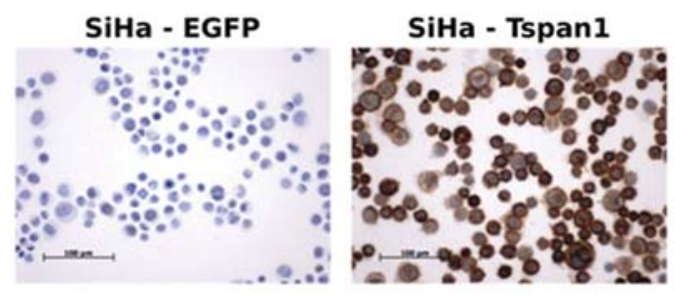

B

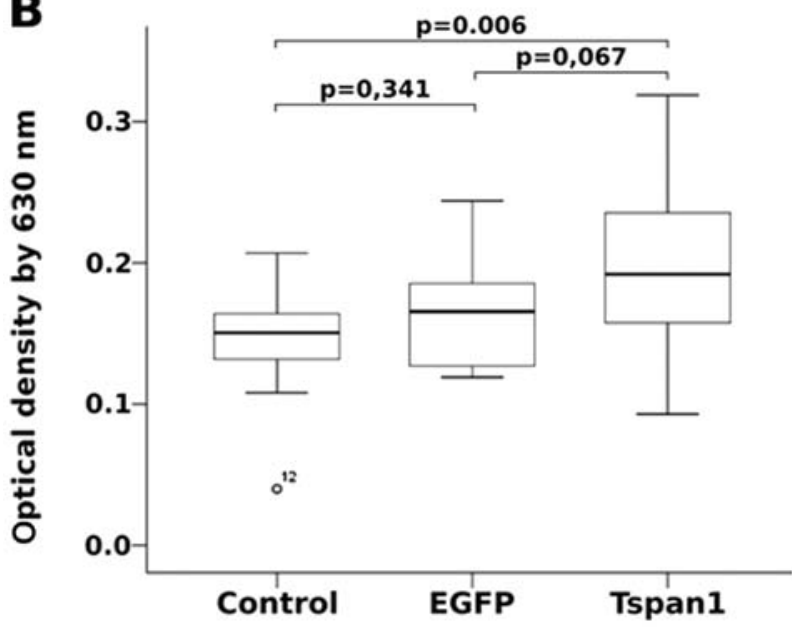

Figure 2. Tetraspanin 1 stimulates invasiveness of cervical cancer cells. (A) Immunocytochemical staining for tetraspanin 1 after adenovirus transduction of SiHa cells. (B) Invasiveness of SiHa cells is significantly increased in the presence of tetraspanin 1 compared to control, native SiHa (control) $(\mathrm{p}=0.006$, Mann-Whitney test). Cell invasion into Matrigel plugs was assessed as described in Materials and methods. Data of four independent experiments with 4-fold determination $(n=16)$ are shown.

cancer tissue. Overall, tetraspanin 1 expression was rather heterogeneous but there was a marked, consistent accumulation of tetraspanin 1 at growth fronts of CIN III and cancer tissues (Fig. 1). In these images dysplastic tumour areas are highlighted by the presence of $\mathrm{p} 16^{\mathrm{INK} 4 \mathrm{a}}$, which accumulates as a consequence of HPV E7 protein action. These data point to a potential participation of tetraspanin 1 in cell proliferation or invasive growth of dysplastic cells in vivo. 

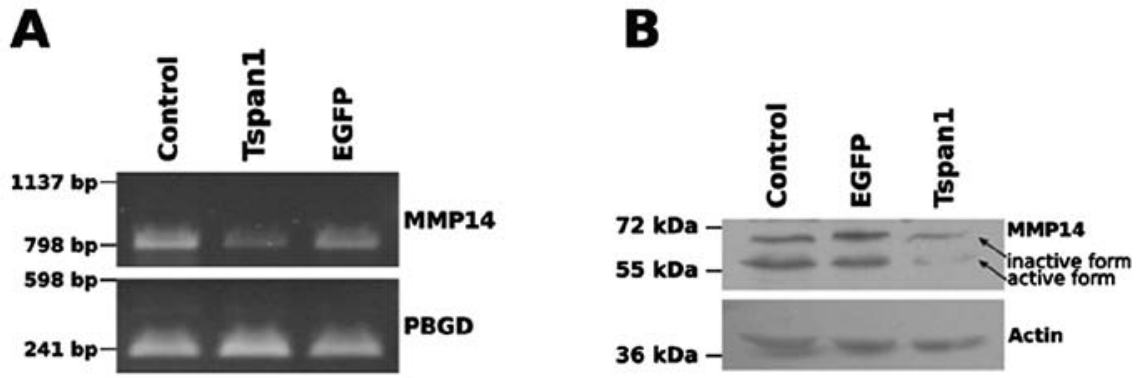

Figure 3. Reduced levels of MMP14 in response to tetraspanin 1 expression. (A) PCR analysis of MMP14 transcript levels from SiHa cells transduced with EGFP or tetraspanin 1. Porphobilinogen deaminase (PBGD) was used as reference gene. (B) Western blot analysis of MMP14 and actin protein levels in cell extracts from the same cell groups. Bands corresponding to the precursor, inactive version and the processed active MMP14 variant are highlighted by arrows.

Tetraspanin 1 enhances cervical cancer cell invasion. The expression of tetraspanin 1 at the growth front of highgrade cervical neoplasias and cervical cancer suggested that tetraspanin 1 might play a role in tissue invasion. To test this hypothesis we analysed the invasive potential of 2 cervical carcinoma cell lines in the presence and absence of tetraspanin 1. The cervical carcinoma cell lines SiHa and HeLa, which are devoid of tetraspanin 1 (Fig. 2A and data not shown), were transduced with an adenovirus driving tetraspanin 1 expression (Fig. 2A). As shown in Fig. 2B tetraspanin 1 significantly increased the invasion of $\mathrm{SiHa}$ cells through matrigel as compared to untransduced or EGFPtransduced SiHa cells. Virtually the same effect was observed in HeLa cells (data not shown). These findings supported the notion that tetraspanin 1 was involved in the modulation of cell invasion of cervical cancer cells.

Control of MMP expression by tetraspanin 1. Tissue invasion by carcinoma cells involves the aberrant expression of enzymes in the remodelling of the extracellular space. MMPs are often upregulated in transformed cells concomitantly with an increase in their invasive properties (reviewed in ref. 50). To learn whether or not the effect of tetraspanin 1 on cell invasion was due to altered MMP expression we monitored the expression of all 23 known human MMP isoforms in $\mathrm{SiHa}$ cells in the presence/absence of tetraspanin 1 by conventional PCR and at the protein level via western blot analysis of cell extracts (49) (data not shown). The expression screen at the mRNA level revealed a decreased expression of MMP14 [or membrane-type 1 MMP (MT1-MMP)] in SiHa cells expressing tetraspanin 1 (Fig. 3A). Reduced MMP14 expression in response to tetraspanin 1 expression was also detected at the protein level (Fig. 3B) and affected in particular the proteolytically processed, activate form of MMP14 (Fig. 3B). These findings revealed an inhibitory effect of tetraspanin 1 on the expression and activation levels of MMP14. At first sight these results appear counter-intuitive with regard to the enhanced invasiveness of tetraspanin 1 expressing $\mathrm{SiHa}$ cells. Indeed, immunohistochemistry of cervical cancer samples revealed no differences in MMP14 expression between tetraspanin 1-positive and -negative areas (data not shown), suggesting that the down-modulation of MMPs observed in $\mathrm{SiHa}$ cells did not reflect the situation in the native tumour and was therefore probably not relevant to the physiological function of tetraspanin 1 in carcinoma of the cervix. Taken together, we concluded that the increased ability of SiHa cells to invade Matrigel following the forced expression of tetraspanin 1 is unlikely to result from an altered MMP expression pattern in cervical cancer cells.

Tetraspanin 1 does not mediate outside-in integrin signalling to focal adhesion kinase. Beyond the release of MMP activity, cell invasiveness requires the concerted regulation of numerous cell biological processes including the spatiotemporal modulation of integrin-dependent signalling and adhesion. Since other members of the tetraspanin family have been linked to the control of integrin activity in the past (reviewed in ref. 25), we moved on to investigate the effect of tetraspanin 1 overexpression on signalling events downstream of integrins. Focal adhesion kinase (FAK) is a major, pivotal transducer of integrin outside-in signals. Adhesion of SiHa cells to fibronectin or collagen type I or III, but not laminin or the inert, charged polymer poly-L-lysine, lead to an activation of FAK, as illustrated by the increase in the phosphorylation of various key tyrosine residues (Fig. 4). Intriguingly, FAK activation/phosphorylation did not translate into paxillin phosphorylation at Tyr118, a bona fide phosphorylation site of FAK (51), suggesting that in SiHa cells paxillin phosphorylation is under control of factors other than the activation status of FAK. Importantly, overexpression of tetraspanin 1 did not markedly affect the phosphorylation pattern of FAK in adhered cells (Fig. 4), strongly indicating that tetraspanin 1 did not regulate cell invasiveness by modulating or transmitting integrin sparked signals.

Effects of tetraspanin 1 on cell adhesion. Since tetraspanin 1 did not affect FAK phosphorylation in response to matrix adhesion we hypothesized that cell adhesion itself would not be grossly affected by tetraspanin 1 . To test this assert and since aberrant cell adhesion is a hallmark of invasive transformed cells, we measured cell adhesion of $\mathrm{SiHa}$ cells to the extracellular matrix (ECM) proteins fibronectin, laminin and collagen type I and III in dependency of tetraspanin 1 expression. No significant differences in adhesion strength to any of the tested ECM proteins, or to uncoated and polyL-lysine coated dishes, were detected between tetraspanin 1 


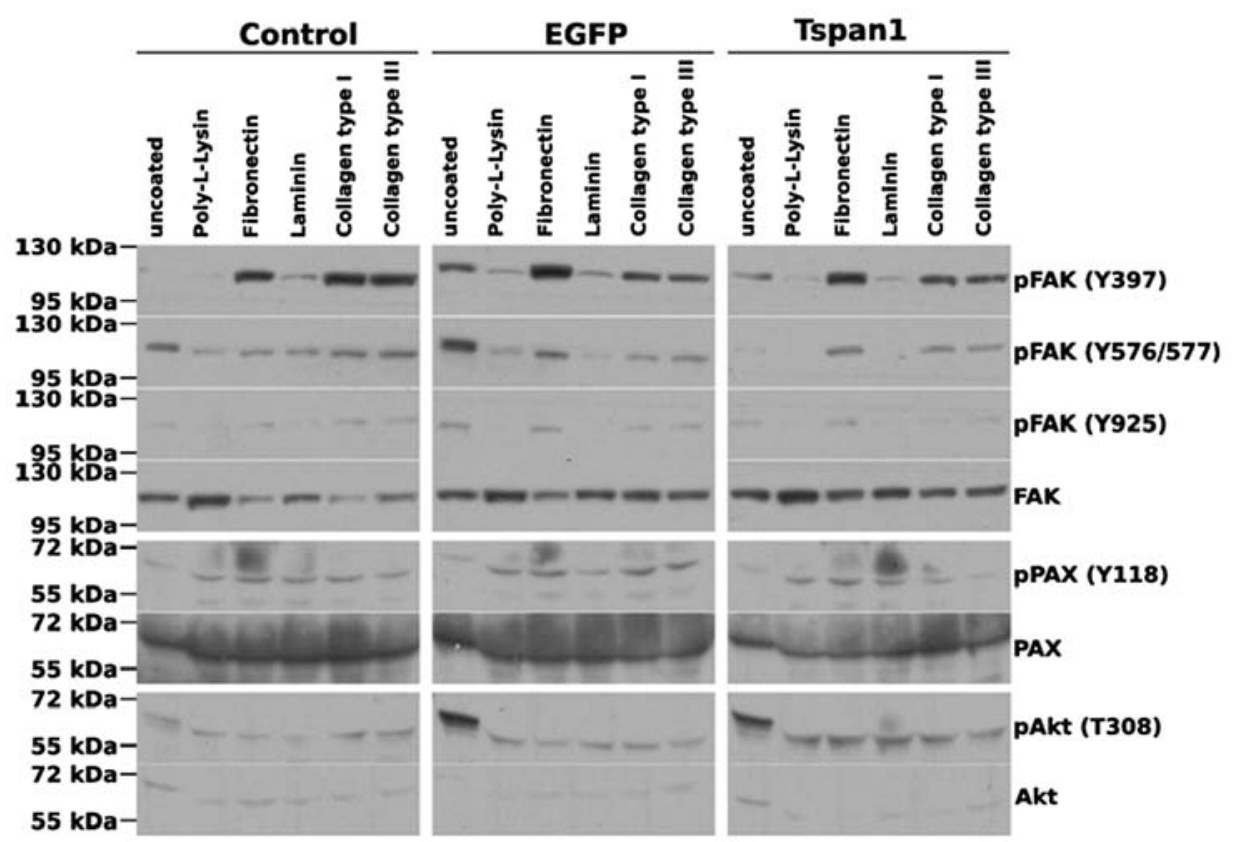

Figure 4. Tetraspanin 1 does not mediate outside-in integrin signalling. SiHa cells transduced with EGFP or tetraspanin 1 expression were plated on different matrix proteins as described in Materials and methods. Cell extracts were prepared and processed for Western blotting with the indicated antibodies. Molecular weight marker bands are indicated on the left. Shown here is a representative experiment out of four independent experiments.

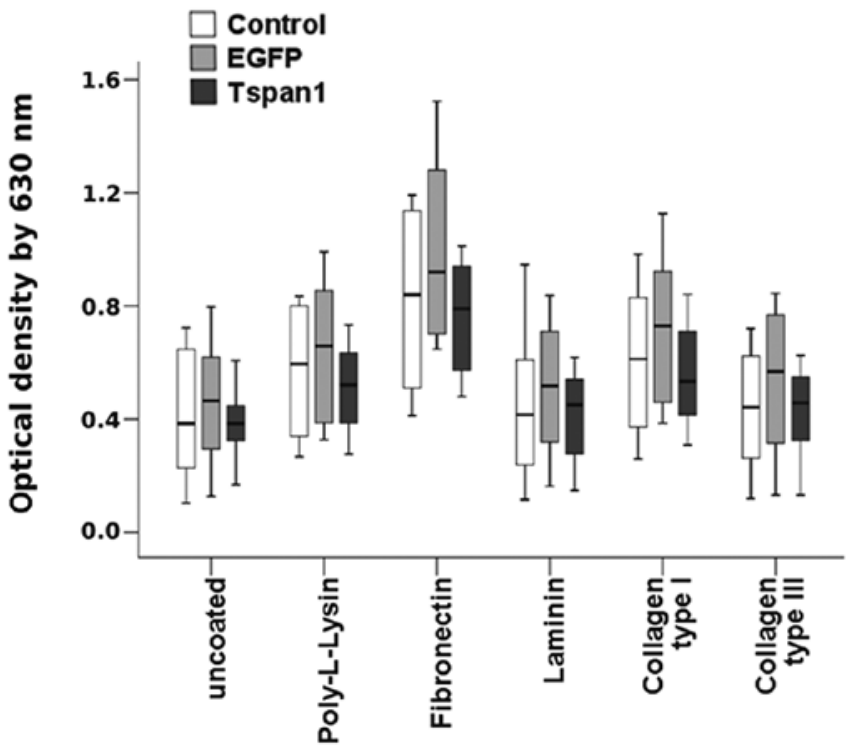

Figure 5. Tetraspanin 1 does not alter adhesion of SiHa cells to ECM proteins SiHa cells transduced with EGFP or tetraspanin 1 and untransduced cells as control were analysed for their adhesion to ECM proteins as described in Materials and methods. Data of four independent experiments with 4-fold determinations $(\mathrm{n}=16)$ are shown.

expressing and untransduced SiHa cells (Fig. 5). These data confirmed the findings shown above and argued against a role of tetraspanin 1 in the control of carcinoma of the cervix cell adhesion to ECM proteins.

Tetraspanin 1 does not affect cell proliferation in culture or in semi-solid media. Previous studies have documented the involvement of tetraspanins in the control of cell proliferation (15). Since proliferation and motility/invasion are intimately intertwined processes we wished to analyse the influence of tetraspanin 1 on tumour cell-specific cell proliferation. To this end, we first assessed growth of SiHa cells in a semi-solid medium, as an indicator of aberrant growth properties. Next, EGFP or tetraspanin 1 transduced $\mathrm{SiHa}$ cells and untransduced cells were seeded in semi-solid media and colony numbers and size distribution were assessed 3 days later. As illustrated in Fig. 6A no significant differences in colony numbers or foci size were observed between native, EGFP or tetraspanin 1 expressing $\mathrm{SiHa}$ cells indicating that tetraspanin 1 did not enhance anchorage-independent proliferation of $\mathrm{SiHa}$ cells. To test whether or not tetraspanin 1 affected proliferation of cervical cancer cells in standard cell culture, native, EGFP or tetraspanin 1 expressing $\mathrm{SiHa}$ cells were subjected to a proliferation assay for $72 \mathrm{~h}$ (data not shown) and for detailed analysis $6 \mathrm{~h}$ (Fig. 6B). Again, no differences in proliferation among the various cell populations could be discerned. These data suggested that tetraspanin 1 does not affect proliferation, either anchorage-dependent or -independent, of cervical cancer cells.

Involvement of tetraspanin 1 in growth factor signalling. Since tetraspanin 1 did not upregulate cervical cancer cell proliferation, we tested whether or not tetraspanin 1 mediated signalling by EGF, a major mitogen of cervical carcinoma cell lines (52-54). EGF induces a very strong activation of Ras and its downstream effector Erk1/2 in these cells $(55,56)$. EGF-triggered activation of Erk1/2 in SiHa (Fig. 7) and HeLa cells (data not shown), monitored by assessing phosphorylation of Erk, was not altered in the presence of tetraspanin 1. Consistently, tetraspanin 1 expression did not affect the extent 
A
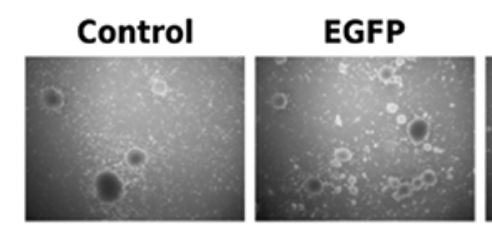

Tspan1

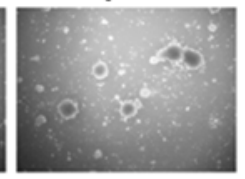

B

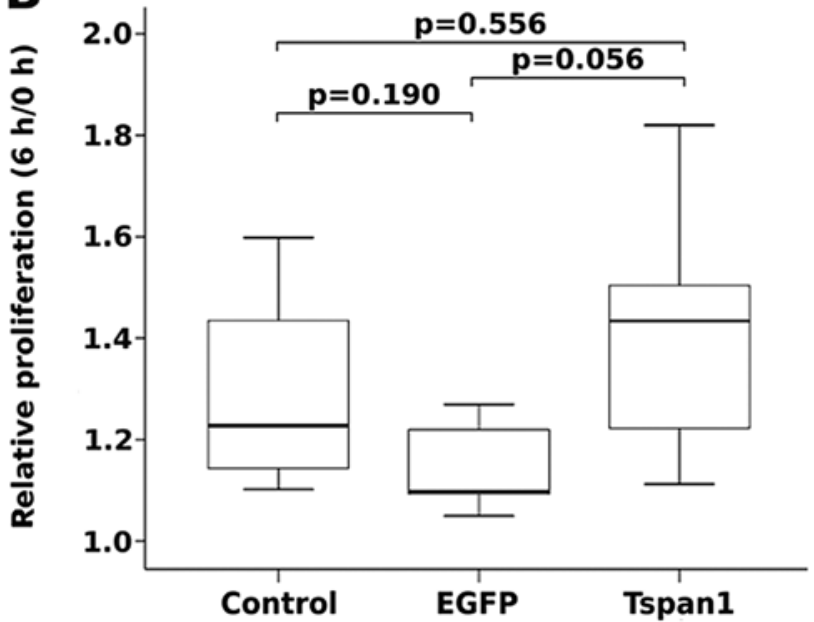

Figure 6. Tetraspanin 1 does not influence proliferation of cervical cancer cells. SiHa cells transduced with EGFP or tetraspanin 1 and untransduced cells were subjected to proliferation assays in semi-solid medium (A) or at standard culture conditions (B) as described in the main text. No difference in colony numbers/size or in liquid culture proliferation $(\mathrm{p}=0.1$, Kruskal-Wallis test) were observed. Normalised data of four independent experiments with duplicates for $\mathrm{SiHa}$ (control) cells $(n=4)$ and five independent experiments with triplicates and duplicates for tetraspanin 1 and EGFP expressing $\mathrm{SiHa}$ cells $(n=5)$ are shown.

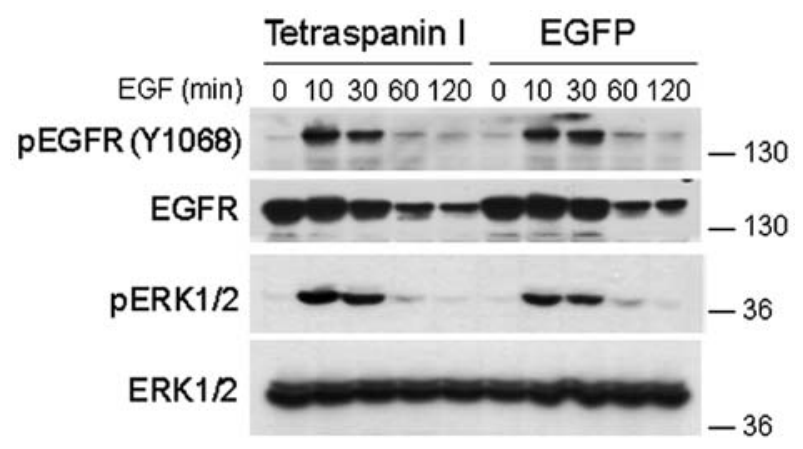

Figure 7. Tetraspanin 1 does not mediate growth factor signalling. SiHa cells transduced with tetraspanin 1 or EGFP as a control were grown in 6-well plates, deprived of serum overnight and challenged with $100 \mathrm{ng} / \mathrm{ml}$ EGF for the indicated periods of time. Cells were lysed and processed for Western blotting with antibodies listed in Table I. Molecular weight markers in kDa are indicated on the right.

of agonist-induced EGF-receptor (EGFR) autophosphorylation, nor did it affect EGFR internalization and degradation, as highlighted by the virtually identical time-dependent reduction of the EGFR signal (Fig. 7). An absence of tetraspanin 1 effects on EGF-signalling was also observed upstream of Erk1/2, at the level of Ras-GTP formation (data not shown). In conclusion, these findings documented that tetraspanin 1 was not involved in receptor-proximal signalling events downstream of the epidermal growth factor receptor.

\section{Discussion}

Tetraspanins are integral components of distinct multiprotein membrane microdomains where they interact with other tetraspanins and other membrane proteins such as major histocompatibility complex (MHC) (57) or integrins (26,58-60) depending on cellular context, tetraspanin isoform and other parameters. These tetraspanin enriched microdomains (TEMs) reportedly play a role in the control of diverse cell biological phenomena such as cell proliferation (15), cell-cell adhesion (61), cell motility and migration $(58,62)$ or signal transduction $(63,64)$. In the present study we investigated the role of tetraspanin 1 in cervical cancer cells. Previously, we have reported a prominent overexpression of tetraspanin 1 in highgrade cervical intraepithelial neoplasia (CIN) and cervical cancer when compared to normal cervical tissue and low-grade CIN. In particular, tetraspanin 1 expression was associated with neoplastic cell proliferation and undifferentiated squamous cell cancers $(40,41)$. In an attempt to determine the role of tetraspanin 1 in cervical cancer, we have used the cervical cancer cell lines SiHa and HeLa, both of which lack detectable tetraspanin 1 expression and studied the consequences of forcing tetraspanin 1 expression for a number of cell biological read-outs. Our data document a role for tetraspanin 1 as a positive regulator of cervical cell motility and invasion, a function that could underlie the observed accumulation of tetraspanin 1 at the growth border of late stage cancers (Fig. 1). This notion is not unprecedented since siRNA-mediated knockdown of tetraspanin 1 expression has previously been observed to severely affect migration and invasion in the A431 and HCT-8 carcinoma lines $(46,47)$, suggesting that tetraspanin 1 might be a global regulator of cell motility in numerous types of cancer. Thus, beyond pointing to a potentially relevant function of tetraspanin 1, these experiments lend support to the possibility of using tetraspanin 1 as a potential marker for disease progression. However, it is likely that tetraspanin 1 may not be the only tetraspanin variant involved in the regulation of cell motility and invasion. Overexpression of the tetraspanin CD151 stimulated migration of HeLa cells (65) and promoted migration and invasion in prostate cancer cell lines LNCaP and PC3 (66). Another tetraspanin family member, CD9, is expressed in normal cervical epithelium and downregulated in cervical cancer, but re-expressed in invasive regions of the tumour (67). Considering all data together, it is tempting to speculate that distinct combinations of tetraspanins could act in concert in distinct cell types to regulate and govern various aspects related to cancer cell motility, adhesion and migration.

In order to understand the mechanism by which tetraspanin 1 regulates the invasive properties of $\mathrm{SiHa}$ and HeLa cells we focused on the expression of MMPs. While we did observe a downregulation of matrix metalloproteinase 14 (MMP14, MT1-MMP) at RNA and protein level in tetraspanin 1 expressing SiHa cells (Fig. 3), immunohistochemical staining of cervical cancer samples did not show any tetraspanin 1dependent changes in MMP14 levels (data not shown), suggesting that changes in MMP expression levels did probably not mediate the effect of tetraspanin 1 on cell motility. A previous report linked the downregulation of MMP14 following the knockdown of tetraspanins CD9, CD81 and TSPAN2 in the breast cancer cell line MCF-7 to a reduced 
invasiveness and colony formation in fibronectin and collagen gels (68), suggesting that the role of MMPs in the control of tetraspanin-dependent cellular process varies depending on the cell type. Interestingly, the downregulation or inhibition of MMP14 is not only expected to dictate the extent of ECM component degradation, but has also been shown to affect, by unknown means, the phosphorylation/activation status of intracellular focal adhesion components FAK and Akt (69). Spatiotemporal modulation of integrin-dependent signalling and adhesion is indeed of paramount importance for correct cell motility and invasion. Our analysis of ECM induced activation of FAK, paxillin and Akt, all critical mediators of outside-in integrin signalling, evidenced no significant differences between cervical carcinoma cells lacking or harbouring tetraspanin 1. Again, this scenario differs from reports in the literature where the knockdown of other tetraspanins, such as CD151 in MDA-MB-231 cells, lead to a reduction of global FAK phosphorylation and to reduced activity of other downstream mediators of integrin signalling like Rac (32). Thus, we hypothesize that the particular mode of action of distinct tetraspanins is likely to vary markedly depending on the tetraspanin species and the cell type under investigation.

For proper cell migration and tissue invasion, cells need to interact with the extracellular matrix via dynamic adhesion complexes and integrin-tetraspanin complexes are components of these structures (70). However, we observed no tetraspanin 1-dependent alteration in adhesion to the matrix polypeptides tested (Fig. 5), which lead us to conclude that the control of cell motility/invasion by tetraspanin 1 was not a consequence of changes in the adhesive properties of the cells. We are currently investigating whether or not tetraspanin 1 affects the GDP/GTP loading status of small GTPases known to be involved in the regulation of cell polarity, cell shape and actin cytoskeleton dynamics. It is worth to note at this point that, as before, the reported effects of tetraspanins on cellular adhesion are rather conflictive and hard to combine in a unifying model framework. For instance, CD9 enhances integrin-dependent adhesion of the colon carcinoma cell line Colo320 (71) and the tetraspanin CD82 stimulates cell adhesion of ovarian cancer cell lines (72). By contrast other carcinoma lines exhibit decreased adhesion to laminin and fibronectin following forced expression of the CD82 $(73,74)$.

Since tetraspanins have also been implicated in the direct or indirect control of cell proliferation $(71,75,76)$ we wished to understand to what extent tetraspanin 1 played a role in anchorage-dependent or independent cell proliferation of cervical cancer cells. The results shown in Fig. 6A argued clearly against a role of tetraspanin 1 as a driver of aberrant cell proliferation in carcinoma of the cervix. Interestingly, other carcinoma cell lines appear to rely heavily on tetraspanin 1 for proliferation $(46,47)$, evidencing once more that tetraspanin 1 is likely to fulfil different functions in different tissues. In line with the lack of an obvious effect on cell growth, tetraspanin 1 expression did not affect signal transduction pathways engaged by EGF (a known mitogen of SiHa and HeLa cells), corroborating that tetraspanin 1 is not a constituent of mitogenic signalling pathways.

In conclusion, in the present study we document an increased invasiveness of cervical cancer cell lines driven by the expression of tetraspanin 1 . At the same time our data argue that tetraspanin 1 is not a genuine signalling mediator of adhesive or mitogenic pathways in cervical carcinoma cells. While the precise molecular mechanisms underlying the control of the cellular motility machinery by tetraspanin 1 remain obscure, our present observations confirm previous findings $(40,41)$ and identify tetraspanin 1 as a potentially useful marker for discriminating neoplasias with low versus high progression potency.

\section{Acknowledgements}

We thank Ute Wittig for excellent technical assistance. We greatly appreciate the advice and support given by HansWalter Zentgraf for generating monoclonal antibodies.

\section{References}

1. GLOBOCAN 2008 (IARC), Section of Cancer Information. International Agency for Research on Cancer; 2008 (accessed $13 / 12 / 2012$ ).

2. Walboomers J, Jacobs M, Manos M, et al: Human papillomavirus is a necessary cause of invasive cervical cancer worldwide. J Pathol 189: 12-19, 1999.

3. de Villiers E, Fauquet C, Broker T, Bernard H and zur Hausen H: Classification of papillomaviruses. Virology 324: 17-27, 2004.

4. zur Hausen H: Papillomaviruses causing cancer: evasion from host-cell control in early events in carcinogenesis. J Natl Cancer Inst 92: 690-698, 2000.

5. Munoz N, Bosch F, de Sanjose S, et al: Epidemiologic classification of human papillomavirus types associated with cervical cancer. N Engl J Med 348: 518-527, 2003.

6. Munoz N, Castellsague X, de Gonzalez AB and Gissmann L: Chapter 1: HPV in the etiology of human cancer. Vaccine 24 (Suppl 3): S3/1-10, 2006.

7. Schwarz E, Freese U, Gissmann L, Mayer W, Roggenbuck B, Stremlau A and Hausen H: Structure and transcription of human papillomavirus sequences in cervical-carcinoma cells. Nature 314: 111-114, 1985

8. Phelps W, Yee C, Munger K and Howley P: The human papillomavirus type-16 E7 gene encodes transactivation and transformation functions similar to those of adenovirus-Ela. Cell 53: 539-547, 1988

9. Munger K, Phelps W, Bubb V, Howley P and Schlegel R: The E6-gene and E7-gene of the human papillomavirus type-16 together are necessary and sufficient for transformation of primary human keratinocytes. J Virol 63: 4417-4421, 1989.

10. Hawleynelson P, Vousden KH, Hubbert NL, Lowy DR and Schiller JT: HPV16 E6-proteins and E7-proteins cooperate to immortalize human foreskin keratinocytes. EMBO J 8: 3905-3910, 1989

11. Halbert C, Demers G and Galloway D: The E7 gene of human papillomavirus type-16 is sufficient for immortalization of human epithelial cells. J Virol 65: 473-478, 1991.

12. zur Hausen H: Papillomaviruses and cancer: from basic studies to clinical application. Nat Rev Cancer 2: 342-350, 2002.

13. Huang S, Yuan S, Dong M, et al: The phylogenetic analysis of tetraspanins projects the evolution of cell-cell interactions from unicellular to multicellular organisms. Genomics 86: 674-684, 2005.

14. Hotta H, Ross A, Huebner K, et al: Molecular-cloning and characterization of an antigen associated with early stages of melanoma tumor progression. Cancer Res 48: 2955-2962, 1988.

15. Oren R, Takahashi S, Doss C, Levy R and Levy S: Tapa-1, the target of an antiproliferative antibody, defines a new family of transmembrane proteins. Mol Cell Biol 10: 4007-4015, 1990.

16. Boucheix C, Benoit P, Frachet P, Billard M, Worthington R, Gagnon J and Uzan G: Molecular-cloning of the Cd9 antigen - a new family of cell-surface proteins. J Biol Chem 266: 117-122, 1991.

17. Levy S, Nguyen VQ, Andria ML and Takahashi S: Structure and membrane topology of tapa-1. J Biol Chem 266: 14597-14602, 1991.

18. Wright MD and Tomlinson MG: The ins and outs of the transmembrane-4 superfamily. Immunol Today 15: 588-594, 1994. 
19. Maecker HT, Todd SC and Levy S: The tetraspanin superfamily: Molecular facilitators. FASEB J 11: 428-442, 1997.

20. Kitadokoro K, Bordo D, Galli G, et al: CD81 extracellular domain 3D structure: Insight into the tetraspanin superfamily structural motifs. EMBO J 20: 12-18, 2001.

21. Seigneuret M, Delaguillaumie A, Lagaudriere-Gesbert $\mathrm{C}$ and Conjeaud H: Structure of the tetraspanin main extracellular domain - a partially conserved fold with a structurally variable domain insertion. J Biol Chem 276: 40055-40064, 2001.

22. Charrin S, Manie S, Oualid M, Billard M, Boucheix C and Rubinstein E: Differential stability of tetraspanin/tetraspanin interactions: Role of palmitoylation. FEBS Lett 516: 139-144, 2002.

23. Escola JM, Kleijmeer MJ, Stoorvogel W, Griffith JM, Yoshie O and Geuze HJ: Selective enrichment of tetraspan proteins on the internal vesicles of multivesicular endosomes and on exosomes secreted by human B-lymphocytes. J Biol Chem 273 20121-20127, 1998.

24. Wubbolts R, Leckie RS, Veenhuizen PTM, et al: Proteomic and biochemical analyses of human B cell-derived exosomes potential implications for their function and multivesicular body formation. J Biol Chem 278: 10963-10972, 2003.

25. Boucheix C and Rubinstein E: Tetraspanins. Cell Mol Life Sci 58: 1189-1205, 2001

26. Rubinstein E, Le Naour F, Lagaudrière-Gesbert C, Billard M, Conjeaud $\mathrm{H}$ and Boucheix C: CD9, CD63, CD81 and CD82 are components of a surface tetraspan network connected to HLA-DR and VLA integrins. Eur J Immunol 26: 2657-2665, 1996.

27. BerditchevskiF, Odintsova E, Sawada S and GilbertE: Expression of the palmitoylation-deficient CD151 weakens the association of alpha(3)beta(1) integrin with the tetraspanin-enriched microdomains and affects integrin-dependent signaling. J Biol Chem 277: 36991-37000, 2002.

28. Tokuhara $\mathrm{T}$, Hasegawa $\mathrm{H}$, Hattori $\mathrm{N}$, et al: Clinical significance of CD151 gene expression in non-small cell lung cancer. Clin Cancer Res 7: 4109-4114, 2001

29. Ang J, Lijovic M, Ashman LK, Kan K and Frauman AG: CD151 protein expression predicts the clinical outcome of low-grade primary prostate cancer better than histologic grading: a new prognostic indicator? Cancer Epidemiol Biomarkers Prev 13: $1717-1721,2004$

30. Sadej R, Romanska H, Baldwin G, et al: CD151 regulates tumorigenesis by modulating the communication between tumor cells and endothelium. Mol Cancer Res 7: 787-798, 2009.

31. Voss MA, Gordon N, Maloney S, et al: Tetraspanin CD151 is a novel prognostic marker in poor outcome endometrial cancer. $\mathrm{Br}$ J Cancer 104: 1611-1618, 2011.

32. Yang XH, Richardson AL, Torres-Arzayus MI, et al: CD151 accelerates breast cancer by regulating alpha(6) integrin function, signaling and molecular organization. Cancer Res 68: 3204-3213, 2008.

33. Ikeyama S, Koyama M, Yamaoko M, Sasada R and Miyake M: Suppression of cell motility and metastasis by transfection with human motility-related protein (mrp-1/cd9) dna. J Exp Med 177: 1231-1237, 1993.

34. Miyake M, Nakano K, Ieki Y, et al: Motility related protein-1 (mrp-1/cd9) expression - inverse correlation with metastases in breast cancer. Cancer Res 55: 4127-4131, 1995.

35. Miyake M, Nakano K, Itoi S, Koh T and Taki T: Motility-related protein-1 (MRP-1/CD9) reduction as a factor of poor prognosis in breast cancer. Cancer Res 56: 1244-1249, 1996.

36. Higashiyama M, Taki T, Ieki Y, et al: Reduced motility related protein-1 (mrp-1/cd9) gene-expression as a factor of poor-prognosis in non-small-cell lung cancer. Cancer Res 55: 6040-6044. 1995.

37. Pileri P, Uematsu Y, Campagnoli S, et al: Binding of hepatitis $\mathrm{C}$ virus to CD81. Science 282: 938-941, 1998.

38. Nydegger S, Khurana S, Krementsov DN, Foti M and Thali M: Mapping of tetraspanin-enriched microdomains that can function as gateways for HIV-1. J Cell Biol 173: 795-807, 2006.

39. Spoden G, Freitag K, Husmann M, Boller K, Sapp M, Lambert C and Florin L: Clathrin- and caveolin-independent entry of human papillomavirus type 16-involvement of tetraspanin-enriched microdomains (TEMs). Plos One 3: e3313, 2008.

40. Nees M, van Wijngaarden E, Bakos E, Schneider A and Durst M: Identification of novel molecular markers which correlate with HPV-induced tumor progression. Oncogene 16: 2447-2458, 1998.
41. Wollscheid V, Kuhne-Heid R, Stein I, Jansen L, Kollner S, Schneider A and Durst M: Identification of a new proliferationassociated protein NET-1/C4.8 characteristic for a subset of high-grade cervical intraepithelial neoplasia and cervical carcinomas. Int J Cancer 99: 771-775, 2002.

42. Chen L, Wang Z, Zhan X, Li D, Zhu Y and Zhu J: Association of NET-1 gene expression with human hepatocellular carcinoma. Int J Surg Pathol 15: 346-353, 2007.

43. Chen L, Li X, Wang G, Wang Y, Zhu Y and Zhu J: Clinicopathological significance of overexpression of TSPAN1, K167 and CD34 in gastric carcinoma. Tumori 94: 531-538, 2008.

44. Chen L, Zhu Y, Zhang X, et al: TSPAN1 protein expression: a significant prognostic indicator for patients with colorectal adenocarcinoma. World J Gastroenterol 15: 2270-2276, 2009.

45. Scholz C, Kurzeder C, Koretz K, Windisch J, Kreienberg R, Sauer G and Deissler H: Tspan-1 is a tetraspanin preferentially expressed by mucinous and endometrioid subtypes of human ovarian carcinomas. Cancer Lett 275: 198-203, 2009.

46. Chen L, Yuan D, Zhao R, Li H and Zhu J: Suppression of TSPAN1 by RNA interference inhibits proliferation and invasion of colon cancer cells in vitro. Tumori 96: 744-750, 2010.

47. Chen L, Zhu Y, Li H, et al: Knockdown of TSPAN1 by RNA silencing and antisense technique inhibits proliferation and infiltration of human skin squamous carcinoma cells. Tumori 96: 289-295, 2010.

48. Saito K, Oku T, Ata N, Miyashiro H, Hattori M and Saiki I: A modified and convenient method for assessing tumor cell invasion and migration and its application to screening for inhibitors. Biol Pharm Bull 20: 345-348, 1997.

49. Koehrmann A, Kammerer U, Kapp M, Dietl J and Anacker J: Expression of matrix metalloproteinases (MMPs) in primary human breast cancer and breast cancer cell lines: New findings and review of the literature. BMC Cancer 9: 188, 2009.

50. Stamenkovic I: Matrix metalloproteinases in tumor invasion and metastasis. Semin Cancer Biol 10: 415-433, 2000.

51. Bellis SL, Miller JT and Turner CE: Characterization of tyrosine phosphorylation of paxillin in vitro by focal adhesion kinase. J Biol Chem 270: 17437-17441, 1995.

52. Ueda M, Ueki M, Terai Y, Morimoto A, Fujii H, Yoshizawa K and Yanagihara T: Stimulatory effects of EGF and TGF-alpha on invasive activity and 5'-deoxy-5-fluorouridine sensitivity in uterine cervical-carcinoma SKG-IIIb cells. Int J Cancer 72: 1027-1033, 1997

53. Ueda M, Fujii H, Yoshizawa K, Terai Y, Kumagai K, Ueki K and Ueki M: Effects of EGF and TGF-alpha on invasion and proteinase expression of uterine cervical adenocarcinoma OMC-4 cells. Invasion Metastasis 18: 176-183, 1998.

54. Narayanan R, Kim HN, Narayanan NK, Nargi D and Narayanan B: Epidermal growth factor-stimulated human cervical cancer cell growth is associated with EGFR and cyclin D1 activation, independent of COX-2 expression levels. Int J Oncol 40: 13-20, 2012.

55. Beeser A, Jaffer ZM, Hofmann C and Chernoff J: Role of group A p21-activated kinases in activation of extracellular-regulated kinase by growth factors. J Biol Chem 280: 36609-36615, 2005.

56. Antonyak MA, Li B, Regan AD, Feng Q, Dusaban SS and Cerione RA: Tissue transglutaminase is an essential participant in the epidermal growth factor-stimulated signaling pathway leading to cancer cell migration and invasion. J Biol Chem 284: 17914-17925, 2009.

57. Angelisova P, Hilgert I and Horejsi V: Association of four antigens of the tetraspans family (CD37, CD53, TAPA-1, and $\mathrm{R} 2 / \mathrm{C} 33$ ) with MHC class II glycoproteins. Immunogenetics 39: 249-256, 1994.

58. Rubinstein E, Lenaour F, Billard M, Prenant $M$ and Boucheix C: Cd9 antigen is an accessory subunit of the vla integrin complexes. Eur J Immunol 24: 3005-3013, 1994.

59. Berditchevski F, Bazzoni G and Hemler ME: Specific association of Cd63 with the vla-3 and vla-6 integrins. J Biol Chem 270: 17784-17790, 1995.

60. Berditchevski F, Zutter MM and Hemler ME: Characterization of novel complexes on the cell surface between integrins and proteins with 4 transmembrane domains (TM4 proteins). Mol Biol Cell 7: 193-207, 1996.

61. Masellissmith A, Jensen GS, Seehafer JG, Slupsky JR and Shaw ARE: Anti-Cd9 monoclonal-antibodies induce homotypic adhesion of pre-B cell-lines by a novel mechanism. J Immunol 144: 1607-1613, 1990.

62. Miyake M, Koyama M, Seno M and Ikeyama S: Identification of the motility-related protein (mrp-1), recognized by monoclonalantibody M31-15, which inhibits cell motility. J Exp Med 174: 1347-1354, 1991. 
63. Olweus J, Lundjohansen F and Horejsi V: Cd53, a protein with 4 membrane-spanning domains, mediates signal-transduction in human monocytes and B-cells. J Immunol 151: 707-716, 1993.

64. Schick MR, Nguyen VQ and Levy S: Anti-tapa-1 antibodies induce protein-tyrosine phosphorylation that is prevented by increasing intracellular thiol levels. J Immunol 151: 1918-1925, 1993.

65. Testa JE, Brooks PC, Lin JM and Quigley JP: Eukaryotic expression cloning with an antimetastatic monoclonal antibody identifies a tetraspanin (PETA-3/CD151) as an effector of human tumor cell migration and metastasis. Cancer Res 59: 3812-3820, 1999.

66. Ang J, Fang B, Ashman LK and Frauman AG: The migration and invasion of human prostate cancer cell lines involves CD151 expression. Oncol Rep 24: 1593-1597, 2010.

67. Sauer G, Windisch J, Kurzeder C, Heilmann V, Kreienberg R and Deissler H: Progression of cervical carcinomas is associated with down-regulation of CD9 but strong local re-expression at sites of transendothelial invasion. Clin Cancer Res 9: 6426-6431, 2003.

68. Lafleur MA, Xu D and Hemler ME: Tetraspanin proteins regulate membrane type-1 matrix metalloproteinase-dependent pericellular proteolysis. Mol Biol Cell 20: 2030-2040, 2009.

69. Takino T, Watanabe Y, Matsui M, Miyamori H, Kudo T, Seiki M and Sato $\mathrm{H}$ : Membrane-type 1 matrix metalloproteinase modulates focal adhesion stability and cell migration. Exp Cell Res 312: 1381-1389, 2006.
70. Berditchevski $\mathrm{F}$ and Odintsova E: Characterization of integrintetraspanin adhesion complexes: Role of tetraspanins in integrin signaling. J Cell Biol 146: 477-492, 1999.

71. Ovalle S, Gutierrez-Lopez MD, Olma N, et al: The tetraspanin CD9 inhibits the proliferation and tumorigenicity of human colon carcinoma cells. Int J Cancer 121: 2140-2152, 2007.

72. Ruseva Z, Geiger PXC, Hutzler P, et al: Tumor suppressor KAI1 affects integrin alphavbeta3-mediated ovarian cancer cell adhesion, motility, and proliferation. Exp Cell Res 315: 1759-1771, 2009.

73. He B, Liu L, Cook GA, Grgurevich S, Jennings LK and Zhang XA: Tetraspanin CD82 attenuates cellular morphogenesis through down-regulating integrin alpha 6-mediated cell adhesion. J Biol Chem 280: 3346-3354, 2005.

74. Lee H, Park I, Byun H, Jeoung D, Kim Y and Lee H: Metastasis suppressor KAI1/CD82 attenuates the matrix adhesion of human prostate cancer cells by suppressing fibronectin expression and beta(1) integrin activation. Cell Physiol Biochem 27: 575-586, 2011.

75. Murayama Y, Miyagawa JI, Oritani K, et al: CD9-mediated activation of the $\mathrm{p} 46 \mathrm{shc}$ isoform leads to apoptosis in cancer cells. J Cell Sci 117: 3379-3388, 2004.

76. Ko E, Lee IY, Cheon IS, et al: Monoclonal antibody to CD9 inhibits platelet-induced human endothelial cell proliferation. Mol Cells 22: 70-77, 2006. 